\title{
Clorinda Matto de Turner y Nísia Floresta. Mujeres precursoras del indigenismo en América Latina
}

\section{Clorinda Matto de Turner and Nísia Floresta: Pioneer Female Writers of Indigenism in Latin America}

\section{Clorinda Matto de Turner e Nísia Floresta. Mulheres precursoras da indigenismo na América Latina}

\author{
Regina Simon da Silva \\ Universidade Federal do Rio Grande do Norte (UFRN), Brasil \\ Recibido 14/08/2014 • Aprobado 30/09/2014 \\ DOI: http://dx.doi.org/10.15359/tdna.31-57.2
}

\section{Resumen}

En este texto examino la novela Aves sin nido (1889) de la peruana Clorinda Matto de Turner, reconocida por los críticos como precursora del indigenismo latinoamericano, y el poema A lágrima de um Caeté (1849) de la brasileña Nísia Floresta, e identifico la obra de esta última como precursora del indigenismo en Brasil, anterior a la obra de Matto de Turner. Asimismo, propongo a Floresta como una de las primeras escritoras que desarrolló un abordaje de carácter indigenista latinoamericano, con el fin de rescatar una

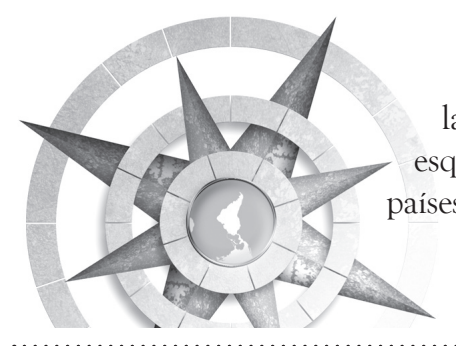

obra olvidada por el canon literario en Brasil y de aproximar a los países que tienen lo indígena como tema literario.

Palabras claves: Nísia Floresta, Clorinda Matto de Turner, indigenismo, literaturas femeninas latinoamericanas, A lágrima de um Caeté, Aves sin nido, Temas de Nuestra América

\section{Resumo}

Neste texto examino o romance Aves sin nido (1889) da peruana Clorinda Matto de Turner, reconhecida pelos críticos como precursora do indigenismo latino-americano, e o poema A lágrima de um Caeté (1849) da brasileira Nísia Floresta, e identifico a obra desta última como precursora do indigenismo no Brasil, anterior à obra de Matto de Turner. Além disso, proponho a Floresta como uma das primeiras escritoras que devolveram uma abordagem de caráter indigenista latino-americano, com o objetivo de resgatar uma obra esquecida pelo cânone literário no Brasil e de aproximar os países que têm o indígena como tema literário. 
Palavras-chave: Nísia Floresta, Clorinda Matto de Turner, indigenismo, literaturas femininas latino-americanas, A lágrima de um Caeté, Aves sin nido, Temas de Nossa América

\section{Abstract}

In this paper I examine the novel Aves sin nido (1889), written by Peruvian author Clorinda Matto de Turner, recognized by the critics as a precursor of Latin American indigenism, as well as the poem $A$ lágrima de um Caeté [A tear of a Caeté] (1849) of the Brazilian writer Nísia Floresta. I suggest that the Floresta's work is a precursor of indigenism in Brazil, even before the work of Matto de Turner. I also propose Floresta as one of the first writers who developed an approach to Latin American indigenism as a way to recover her work forgotten by the literary canon in Brazil and, to bring these countries closer since they have the indigenous topic as a literary theme.

Keywords: Nisia Floresta, Clorinda Matto de Turner, Indigenism, Latin American Female Literatures, A lágrima de um Caeté, [A Tear of a Caeté], Birds without a Nest, Temas de Nuestra América.

\section{Introducción}

La intención de realizar este estudio surgió mientras desarrollaba una investigación acerca de la literatura producida por mujeres en el siglo XIX.
En aquella ocasión hice un análisis comparado entre las escritoras Flora Tristán y Nísia Floresta, ${ }^{1}$ motivado por la historia de vida de ambas mujeres. Tristán ya formaba parte de mis estudios, pero a Floresta acababa de conocerla. Esto debido no solo a los pocos estudios dedicados a las mujeres, sino también a la poca receptividad de los escritos nisianos por parte de la crítica en Brasil.

Sin embargo, se debe valorar el esfuerzo desarrollado por Constância Lima Duarte, pues a partir de la publicación de su tesis doctoral Nísia Floresta vida e obra, en 1995, se despertó el interés por traducir al portugués sus obras aún inéditas en este idioma o por reimprimir viejas ediciones, lo que permitió la circulación de sus textos entre los investigadores.

Prosiguiendo con los estudios comparados, que según Schwartz (2008) son una forma de buscar la integración histórico-geográfica y la demolición del «muro de Tordesillas» que siempre aisló a Brasil de la América Hispánica, me propongo examinar la novela Aves sin nido (1889) de la peruana Clorinda Matto de Turner, reconocida por los críticos como precursora del indigenismo latinoamericano, y el

1 Flora Tristán y Nísia Floresta: historias que se cruzan, pensamientos que se completan, es el título de la ponencia presentada en el I Congreso Internacional Las Mujeres en los Procesos de Independencia de América Latina, realizado el 21, 22 y 23 de agosto de 2013, en Lima, Perú.

\section{Clorinda Matto de Turner y Nísia Floresta... Regina Simon da Silva}


poema A lágrima de um Caeté (1849) de la brasileña Nísia Floresta, que en el prólogo a la cuarta edición (1997) Lima Duarte identificó como una obra pionera del indigenismo en Brasil, es decir, anterior a la obra de Matto de Turner.

Se plantea, entonces, la inclusión de la obra de Floresta como uno de los primeros abordajes de carácter indigenista latinoamericano, con el fin de rescatar una obra olvidada por el canon literario en Brasil y de aproximar a los países que tienen lo indígena como tema literario, guardando, sin embargo, las debidas diferencias respecto a las culturas autóctonas existentes en América y los procesos de colonización aquí emprendidos.

\section{Consideraciones acerca del indigenismo y del indianismo}

El siglo XIX significó para los países americanos un momento de profunda reflexión político-cultural en busca de elementos que posibilitaran la construcción identitaria de la nación. El elemento indígena se encontraba en las discusiones y en el discurso de los principales pensadores y literatos de los países que componían la América Latina durante los procesos de independencia de las colonias, razón por la que se pretende buscar un diálogo entre las Américas española y portuguesa. Sin embargo, es importante señalar que, mientras la independencia de las colonias españolas resultó en la formación de Repúblicas independientes, el proceso de emancipación política de Brasil culminó con la institución del Imperio de Brasil, régimen monárquico que existió entre 1822 y 1889, cuando se proclamó la República.

Resguardada esta peculiaridad, puedo decir que esto no significó un vaciamiento respecto a los discursos nacionalistas, ya que al ser un tema en los textos que comprenden el periodo colonial, al Imperio le correspondía convertir al indio en símbolo de nacionalidad, tal como se puede verificar en la siguiente cita:

O século XIX traz como marca um amplo debate acerca da presença indigena, em busca de soluções para o que se denominava à época "problema índio". Ideias circulavam no âmbito cotidiano da população e ao mesmo tempo, entre políticos e intelectuais oitocentistas e, nesse contexto, o desafio lançado para a inserção de um "outro" no projeto que se definia para a nação toma as páginas das Revistas do Instituto Histórico e Geográfico Brasileiro² desde a sua

2 N.de traducción: La publicación del Instituto Histórico e Geográfico Brasileiro(IHGB) fue establecida por el cura Januário da Cunha Barboza, secretario inaugural del Instituto, en 1839, en Río de Janeiro. Tenía como fin no sólo enseñar y difundir el conocimiento, sino que también el de formar una visión histórica, que se dedicase a la exaltación de la patria, atendiendo a las demandas de una clase política con ganas de formar y consolidar su proyecto de Estado nacional y la identidad brasileña. Los investigadores de entonces creían que la humanidad estaba inserida en un proceso de desarrollo histórico, que partía desde las 
primeira edição, em 1839, um ano após a criação do instituto. Presença marcante no imaginário da nação, $\mathrm{o}$ indígena permeia nossa cultura política, ora como ícone romântico e idealizado, ora como sinônimo de atraso e empecilho ao progresso (Portela, 2011, p. 60).

En la cita textual anterior, a partir de los fragmentos «soluciones para el problema del indio», "cultura política» e «ícono romántico e idealizado», se evidencia que el indio formaba parte del discurso, tanto de los proyectos políticos que se gestaban (no siempre con intereses que beneficiaban a los indios) como de la literatura, dificultando la conceptualización del tema y la asimilación por parte de los investigadores, que interpretan cada uno a su modo y producen una avalancha de pensamientos de difícil consenso.

Como punto de partida, me apoyo en las palabras de Rodríguez-Luis, que planteó la diferenciación entre los sustantivos indigenismo e indianismo:

sociedades primitivas, indios y negros, hasta alcanzar la cumbre de la civilización, representada por los europeos blancos. Fieles a esa teoría, los miembros del IHGB comenzaron una compilación de documentos históricos en todo el territorio del país. Invariablemente, el análisis de esas fuentes indicaba el estado de barbarie en el que vivían las tribus indigenas, al mismo tiempo en que destacaba el papel redentor del hombre blanco, que vino a salvar a los autóctonos del "obscurantismo" en el que vivían. [Traducción a cargo de la autora del artículo] Fuente original recuperada de:: http:// www.museudavida.fiocruz.br/brasiliana.
Se entiende por indigenismo el estudio sociológico y antropológico del indígena iberoamericano, estudio que se proyecta en el plano político hacia la reivindicación social y económica de aquél. Al decir «indígena» queda establecido que se trata en el indigenismo del habitante original del continente y no del «indio» que creyó hallar Colón. Es el «indio», en lugar del indígena, el objeto de la literatura indianista que en la segunda mitad del XIX idealiza al «buen salvaje» americano como parte del proyecto romántico de definir nuestra especificidad frente a Europa, pero principalmente para compensar así la opresión que el indígena venía sufriendo desde la conquista (1990, p. 41).

Estas acepciones son semejantes, en gran medida, a las que se establecen en Brasil de indigenismo, entendido como política de poder de los Estados para conocer y resolver los problemas que enfrentan los pueblos indígenas, con el fin de integrarlos a la sociedad, e indianismo, vinculado a la producción literaria de la primera fase del Romanticismo que tenía al indio y a las costumbres indígenas como tema central.

Para Rodríguez-Luis (1990) esta distinción es necesaria porque las novelas y poemas indianistas se vinculan con un plano estrictamente individual que ayuda a evitar la confrontación de la temática de la 
explotación del indígena, lo que el liberalismo romántico no podía acusar, pues carecía de alternativas. Sobre esta cuestión volveré al analizar la obra A lágrima de um Caeté de Nísia Floresta.

Esta distinción se percibe en la actualidad por medio de la investigación de estudiosos del tema, cito dos ejemplos: Para além do "caráter ou qualidade de indígena": uma história do conceito de indigenismo no Brasil (2011) de Cristiane de Assis Portela y Utopia e agonia - o indianismo de Gonçalves Dias (2014) de Andrey Pereira de Oliveira.

A manera de referencia, el trabajo de Assis Portela, consiste en una historización del concepto de indigenismo, buscando en la intelectualidad del siglo XIX los elementos que le dieron significado al término. Entre los textos analizados se encuentran las producciones literarias de la época. La autora argumentó que el concepto de indigenismo, según se concibe hoy, conlleva el peso semántico y las marcas de las oposiciones construidas históricamente en torno al tema, realizando un largo análisis en busca de lo que ella llamó noción de indigenismo en la historiografía del siglo XIX. En el caso del de Pereira Oliveira, se trató de la tesis de doctorado presentada en el Programa de Posgrado en Literatura, Universidad Federal de Paraiba - UFPB. Es un estudio que reflexiona acerca de la poesía indianista de Gonçalves Dias (1823-1864), uno de los mayores representantes del romanticismo brasileño, contemporáneo de Nísia Floresta.

El primer trabajo puso énfasis en el carácter sociológico e histórico del tema, mientras que el segundo privilegió el análisis crítico del texto literario. Sin embargo, el objetivo en ambos delimitó el corpus para el análisis de la obra de Gonçalves Dias. Portela se enfocó en dos trabajos: Meditação, obra publicada parcialmente en la Revista Guanabara, entre los años 1849 y 1856 , y publicada integralmente en 1909, que reúne reflexiones acerca de la nacionalidad brasileña, y O Brasil e a Oceania, estudio comparado por encargo de por Don Pedro II, presentado en sesión solemne del IHGB, en 1849 y publicada posteriormente de 1867. Oliveira analizó la obra Poesias americanas ${ }^{3}$ (1998) libro cargado de elementos locales, motivado por el ambiente nacionalista en el que estaba envuelto.

Como es posible observar, la distinción entre indianismo e indigenismo en Brasil queda bien establecida, independientemente de cómo sean presentados los temas

3 Los poemas compilados bajo el título Poesías Americanas se encuentran distribuidos en los libros de Gonçalves Dias: Primeiros cantos (1847); Segundos cantos (1848); Últimos cantos (1851); además del épico incompleto "Os Timbiras" (1857) y algunos reunidos en las Obras póstumas (1869). (Oliveira, 2014, p. 14-5) 
del indio y de la ideología defendida por el autor que da vida al texto. En Hispanoamérica, considero, el movimiento presenta mayor grado de complejidad, no solo con relación al tema, sino también al número de investigaciones y de obras producidas y al complejo cultural representado por las culturas precolombinas, que en un primer momento se acercaba a lo visto anteriormente:

Desde el momento de la Independencia, es cierto, el mestizo americano inició la búsqueda de sí mismo. Rotas violentamente las ligas con España y con el sistema de valores que ésta representaba creyó ver en la vuelta al pasado indio el punto en que fundar su propia afirmación. Nació así un romántico movimiento indianista que pretendió reconstruir en México y en Perú el imperio del incario y el de los antiguos aztecas, la vieja organización social y aun las religiones precolombinas suplantadas por el cristianismo (Aguirre, 1956, p. 53).

Las producciones literarias de este período son analizadas en el clásico libro de la puertorriqueña Concha Meléndez (1961), La novela indianista en Hispanoamérica 1832-1884 y que, por el período que abarcan, Escajadillo (2014) ha considerado como antecedente del movimiento indigenista. Según Rodríguez-Luis:
Habrá que esperar hasta que se produzcan la denuncia de la explotación del indígena y el planteamiento de la necesidad de su liberación como paso esencial a la re-estructuración política, social y económica de las naciones hispanoamericanas con poblaciones indigenas numerosas para que se empiece a producir una literatura centrada en la verdadera situación del indígena (1990, p. 41).

Es decir, en Hispanoamérica la voz indigenista se aplica a la literatura, con un significado concreto relativo a un movimiento, tendencia o escuela, a diferencia de lo que relaté en el caso de Brasil. Sin embargo, Ángel Rama problematizó la cuestión en Transculturación narrativa en América Latina, justificando el fracaso de los escritores indigenistas en dar vida al indígena:

Inútil subrayar que en ninguna de esas oportunidades habló el indio, sino que hablaron en su nombre, respectivamente, sectores de la sociedad hispánica o criolla o mestiza. Inútil también agregar que en todos los casos, fuera de la convicción puesta en el alegato a favor del indígena, lo que movía principalmente ese discurso eran las propias reivindicaciones de los distintos sectores sociales que las formulaban, sectores minoritarios dentro de cada sociedad, pero dueños de una intensa movilidad social y un bien determinado 
proyecto de progreso social, que engrosaban sus reclamaciones propias con las correspondientes a una multitud que carecía de voz y capacidad para expresar las suyas propias (Rama, 1987, p. 139).

Rama se refirió a las veces que el indio aparecía en la historia de la América conquistada como pieza maestra de una reclamación: en un primer momento, en la literatura misionera de la Conquista; luego en la literatura crítica de la burguesía mercantil, en el período precursor y revolucionario; por tercera vez en el período romántico, como expresión de la larga lamentación con que se acompañaba su destrucción; y por cuarta vez, en el siglo XX, bajo la forma de una demanda que presentaba un nuevo sector social, procedente de los bajos estratos de la clase media, blanca o mestiza. Partiendo de las observaciones de Rama respecto al indígena, Rodríguez-Luis definió el movimiento indigenista como exterior y con un propósito artificial. Sin embargo, "gracias a esa exterioridad, la novela indigenista consigue traducir como una tensión capaz de provocar nuestro entusiasmo por la causa de la reivindicación del indígena» (Rodríguez, 1990, p. 43). Comparto esta opinión de Rodríguez-Luis, pues los cambios no se producen sin darse el primer paso. De ahí la importancia que tienen las obras precursoras: abren el camino para las manifestaciones posteriores y son juzgadas por sus aciertos y fracasos:

Vista en su conjunto a lo largo del medio siglo que va desde la obra de Matto de Turner a la de Alegría, y en relación al proyecto que entraña respecto al indígena, la narrativa indigenista se nos aparece como victoriosa al mismo tiempo que fallida. Victoriosa en cuanto a haber contribuido efectivamente a revelar la explotación del indígena y, por lo tanto, a hacer conscientes a las burguesía de los problemas sociales, lo que redundará en algún alivio de esa explotación (...) Y puesto que la novela indigenista si había conseguido su propósito político de denunciar la explotación del indígena, podía también afirmarse que para ese entonces había concluido su vida histórica ya que nuevas manifestaciones del mismo proyecto no podían avanzar sino sólo repetir la descripción de la situación del indígena (Rodríguez, 1990, p. 44).

\section{Clorinda Matto de Turner y el indi- genismo precursor}

Clorinda Matto de Turner ${ }^{4}$ nació en Cuzco (Perú), en 1854, donde pasó su juventud y probablemente aprendió a

4 Para una biografía simplificada de Clorinda Matto de Turner se sugiere: http://www.alohacriticon. $\mathrm{com} /$ viajeliterario/article $1655 \mathrm{html}$ 
hablar el quechua. Fue influenciada por la producción de Emile Zola y la de Manuel González Prada, además participó en las veladas literarias de Mercedes Cabello de Carbonera. Fue una de las principales cultivadoras de la novela realista latinoamericana de finales del siglo XIX. Vivió en Tinta con su marido inglés hasta el fallecimiento prematuro de este. Trabajó como periodista y dirigió un salón literario en Lima con destacada presencia femenina. Fue una mujer preocupada por la situación social de los indígenas y escribió el clásico libro Aves sin nido (1889), importante novela indigenista en la que denunciaba la situación precaria del indígena. Matto de Turner ha escrito libros como Tradiciones cuzqueñas (18841886), Índole (1891) y Herencia (1893). Además, se ocupó de traducir la Biblia al quechua. Falleció en 1909 en la ciudad de Buenos Aires, a los 54 años.

En el prólogo a la edición de 1994 de Aves sin nido Antonio Cornejo Polar destacó que, tras un temprano y excepcional éxito editorial, la crítica pronto se olvidó de esta obra, o la maltrató dura e injustamente y que solo después de 1934, cuando fue reivindicada por la profesora Concha Meléndez, se volvieron a publicar nuevas ediciones permitiéndole a las generaciones jovenes el contacto con esta novela y la posibilidad de realizar nuevos análisis.

Lostextosanalizados parala producción de este estudio suelen presentar ideas muy similares. Destacan el contexto social y el drama producido en el Perú tras la trágica experiencia de la Guerra del Pacífico (1879-1883), que culminó en el deterioro económico, social, político y ético del país. Sin embargo, Cornejo Polar afirmó que Matto de Turner trató muy pocas veces el tema de manera explícita, pero para él «es obvio que su vocación reformadora se nutre esencialmente de la experiencia de una nación vencida que tiene que encontrar alternativas sociales de reconstrucción» (Cornejo, 1994: p. x).

Cornejo Polar afirmó que Aves sin nido se relaciona con las preocupaciones nacionales del siglo XIX, a saber: en primer lugar, el uso de la vida nacional como argumento; luego, la crítica de los problemas sociales más urgentes; $y$, finalmente, los beneficios que puede extraer la República de obras de esa naturaleza. Según él, con alguna que otra indecisión, Aves sin nido cumple este programa:

La imagen que Aves sin nido ofrece de los indios tiene rasgos marcadamente románticos y de alguna manera se asocia con el estereotipo del «buen salvaje». Los indios son 
alabados por la «encantadora sencillez de sus costumbres», y aparecen siempre como seres inocentes, buenos y candorosos, pero esa misma bondad natural los hace singularmente vulnerables frente a la codicia de los potentados y de las autoridades de la región. Clorinda Matto anota insistentemente, además, las deficiencias educativas que hacen imposible imaginar siquiera el progreso de la que varias veces llama «la desheredada raza» (Cornejo, 1994: p.xiv).

Pienso que esta cita ilustra, de manera significativa, unos motivos claros para la realización de nuevos estudios sobre Aves sin nido, cuestionando el carácter indigenista de la obra de Matto de Turner.

En el artículo "Aves sin nido ¿novela “indigenista”? Tomás Escajadillo (2004) realizó una nueva lectura de la novela, ya que la crítica hispanoamericana se ha interesado en señalar el valor "histórico» que tuvo la novela --con lo que él está de acuerdo- y su importancia como primera novela indigenista -con lo que está en absoluto desacuerdosin realizar un análisis interno de la novela, tarea puesta en práctica por él. Para el desarrollo del trabajo él expuso las tres condiciones indispensables para considerar una obra como indigenista:
La primera, el «sentimiento de reivindicación social», que según él la novela cumple largamente, desde la declaración del «Proemio» (...) hasta la "poética» de sello naturalista que cree ver en la novela un instrumento de alta eficacia para el mejoramiento de la sociedad (...) Aves sin nido no cumple, sin embargo, las otras dos «condiciones» esenciales que debe tener una obra auténticamente «indigenista»: no hay «ruptura con el pasado», pues subsiste una gran cantidad de elementos románticos y, finalmente, no se consigue una «suficiente proximidad» con relación al mundo recreado, pues el indio y el paisaje andinos en esta novela se nos aparece borrosos, lejanos, no individualizados (p. 132).

Es posible observar, a partir de las propuestas de Escajadillo, que cuando Cornejo afirmó «que la imagen que Aves sin nido ofrece de los indios tiene rasgos marcadamente románticos» (1994: 19) apuntaba al no cumplimiento de una de las condiciones esenciales señaladas por Escajadillo. Se puede concluir, entonces, que Aves sin nido no cumple con todas las condiciones de una novela genuinamente indigenista; sin embargo, no ha repetido en su totalidad los modelos románticos del indianismo. Al innovar denunciando la situación real de la vida del indígena 
se puede considerar como precursora del indigenismo en Hispanoamérica.

Nisia Floresta y A lágrima de um Caeté, ¿una obra indianista?

Nísia Floresta nació en 1810 en Floresta, en la entonces Capitanía de Río Grande del Norte, nordeste de Brasil, hija del abogado portugués Dionísio Gonçalves Pinto Lisboa y de la brasileña Antonia Clara Freire. Se casó a los trece años, un casamiento impuesto conforme la mentalidad de la época, pero pasados dos años abandonó al marido y volvió a vivir con sus padres. Más tarde se unió a Manuel Augusto de Faria Rocha, con quien tuvo tres hijos, aunque el primero no sobrevivió. La felicidad al lado del compañero de su elección fue interrumpida por la muerte repentina de este en 1833.

Viajera en su esencia, Floresta vivió en varias regiones de Brasil, como Pernambuco, Porto Alegre y Río de Janeiro, y emprendió su primer viaje a Europa en 1849, donde decidió vivir; volvió a Brasil solamente dos veces y por cortos periodos. Murió en 1885 en Rouen, Francia, a los 75 años.

Desde temprana edad esta brasileña sorprendía por su coraje en la lucha por los derechos de las mujeres y de las clases marginadas, rompiendo las barreras que impedían que las mujeres ocuparan espacios públicos. Publicó su primer libro bajo el sugerente título Direito das mulheres e injustiça dos homens en 1832. Empezó una carrera como educadora en Río de Janeiro, donde mantuvo una escuela para niñas: el colegio Augusto, inaugurado en febrero de 1838.

Dueña de una extensa obra literaria publicada en portugués, francés e italiano, Floresta es poco conocida entre los brasileños, a pesar de la reciente reedición de algunas de sus obras y de la traducción de otras producidas fuera de Brasil.

El poema A lágrima de um Caeté reúne, según Constancia Duarte, las dos mayores tendencias del romanticismo brasileño: la cuestión indígena y las luchas politicas, ambas impregnadas de una fuerte carga nacionalista. La dinámica de esa unión resume el argumento del poema:

Para realizar esta fusión, la narrativa construye dos tiempos históricos: el de la colonización y el del Imperio. En el primero, el protagonista es el Caeté (representando el indio brasileño), y el opresor, el colonizador portugués. En el segundo momento, los protagonistas son los liberales (con destaque para Nunes Machado) y los antagonistas, los hombres del Imperador. En la trayectoria del tiempo pasado para el presente transcurre

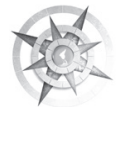


el drama del indio brasileño: de protagonista de la historia a espectador de la derrota liberal y de la suya propia en cuanto civilización ${ }^{5}$ (Duarte, 1997, p. 13).

Así como Matto de Turner, Floresta conoció de cerca el contexto histórico de la época y a los líderes revolucionarios de la Revolução Praiera, movimiento de carácter liberal y federalista que estalló durante el segundo reinado de D. Pedro II, en la provincia de Pernambuco entre los años $\underline{1848}$ y $\underline{1850}$, con quienes ella compartía los ideales de libertad. El hecho motivó a Floresta a escribir este poema, que se traduce en un cambio de actitud de la autora, reconocible en la emoción y el involucramiento con los que se expresa en los versos.

En varios momentos la voz poética le da la palabra al indio y defiende su derecho de luchar por la libertad contra el colonialismo extranjero; se identifica con el Caeté y se muestra sensible con su sufrimiento, cumpliendo la primera de las condiciones propuestas por Escajadillo. A continuación cito un fragmento de la obra:

De repente troar ao longe ouviu-se

Da artilharia o fogo...e de milhares

De peitos Brasileiros sai o brado,

Simulando o trovão, que o raio manda

5 Traducción del texto a cargo del autor del artículo.
- Eia! Avante! Guerreiros libertemos

A terra dos Caetés, a terra nossa!

E a tempestade por Deus fulminada

Sobre um povo ingrato, que Ele amaldiçoa

Varão denodado às fileiras voa

Dos filhos que a Pátria querem libertada!

Dos bravos Caetés se diz descendente, Sua triste raça jurou de vingar...

Desde lá do berço aprendeu a amar

O triste oprimido, dele defendente 6 (Floresta, 1997, p. 45).

En cuanto a la segunda condición, la ruptura con el pasado, probablemente la autora no tuvo esa intención, principalmente porque ese texto fue publicado en 1849, en la primera etapa del romanticismo brasileño. Sin embargo, se puede observar que el poema no se asemeja a otras obras del mismo período: introduce personajes reales de la historia de Brasil y juega

6 N. de T.: De repente tronar a lo lejos se oyó / De la artillería el fuego... y de millones / De senos brasileños sale el clamor, / Simulando el trueno, que envía el rayo / ¡Arre! ¡Adelante! Guerreros libertemos / ¡La tierra de los Caetés, la tierra nuestra! // Y la tempestad por Dios enviada / Sobre un pueblo ingrato, que Él maldice / Varón valiente a las filas vuela $/$ iDe los hijos que la Patria quieren independizada! // De los bravos Caetés se dice sucesor, / Su triste raza juró vengarse... / Desde la cuna aprendió a amar / El triste oprimido, de él defensor. 
con el anacronismo, uniendo hechos que el tiempo real separa. Por todo esto, creo que Floresta, aunque sin intención de ello, produjo cambios importantes que la alejaron de los otros textos de la misma época.

La tercera condición propuesta por Escajadillo es la proximidad con relación al mundo recreado. Cabe tomar en cuenta que A lágrima de um Caeté reproduce los anhelos libertarios del grupo liderado por Nunes Machado, que murió el 02 de febrero de 1849. En mayo del mismo año salió la primera edición y en el Avant-Propos la autora se refirió a la dificultad encontrada para la publicación del libro, lo que sugiere una posible censura de esta edición. Creo que también esta condición se cumple en la obra de Floresta:

O infeliz Caeté, a pesar de ter chegado a esta corte no mês de fevereiro logo depois da revolta dos Rebeldes em Pernambuco, é somente agora que lhe permitiram aparecer, e isto depois de o terem feito passar por mil torturas inquisitoriais!... Graças à benfazeja mão, que o fez renascer, qual Fênix, das cinzas a que o haviam ou queiram reduzir!? (Floresta, 1997, p. 35).

7 N. de T.: El infeliz Caeté, a pesar de haber llegado a esta corte en el mes de febrero tras la insurrección de los Rebeldes en Pernambuco, solo ahora es que le permitieron aparecer, y esto luego de haberlo hecho pasar por mil torturas inquisitoriales!... Gracias a la mano bienhechora, que lo hizo renacer, cual Fénix, de las cenizas a que lo habían o querían reducir!
En conclusión, la obra Aves sin nido de Clorinda Matto de Turner (considerada por gran parte de la crítica como una obra de carácter indigenista), según los criterios propuestos por Escajadillo, no cumple con todos los requisitos, lo que la sitúa como precursora de este movimiento; por otro lado, A lágrima de um Caeté de Nísia Floresta, entendida por la crítica como una obra indianista, al pasar por el mismo análisis legitima el cumplimiento de todos los requisitos, lo que la define como una obra indigenista.

Sin embargo, pienso que A lágrima de un Caeté aún mantiene fuertes vínculos con el romanticismo y el proyecto romántico del cual forma parte. Además, cuenta con muchas diferencias con relación a las novelas indigenistas producidas a partir del siglo XX, lo que también le confiere la denominación de precursora del indigenismo en América Latina, es decir, anterior a la obra de la peruana.

\section{Referencias bibliográficas}

Aguirre, G. (1956). Indigenismo y mestizaje: una polaridad bio-cultural. Revista Municipal de Artes y letras, año IV(1718), 51-64.

Cornejo, A. (1994). Aves sin nido como alegoría nacional. Prólogo. En Aves sin nido de C. Matto de Turner (ix-xxvii). Venezuela: Biblioteca Ayacucho.

Dias, G. (1867). O Brasil e a Oceania. Rio de Janeiro: H. Garnier.

\section{Clorinda Matto de Turner y Nísia Floresta...

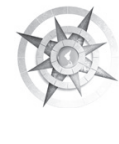


. (1909). Meditação. Rio de Janeiro: H. Garnier.

. (1998). Poesia e prosa completas. (organização de Alexei Bueno). Rio de Janeiro: Nova Aguilar.

Duarte, C. (1997). A lágrima de um Caeté: uma nova página do indianismo brasileiro. Em Floresta, N., A lágrima de um Caeté (4 ed.), (pp.7-31), Natal, Brasil: Fundação José Augusto.

Escajadillo, T. (2014). Aves sin nido ¿novela «indigenista»? Revista de crítica literaria latinoamericana, año XXX(59), 131-154.

Floresta, N. (1997). A lágrima de um Caeté (4 ed.). Natal, Brasil: Fundação José Augusto.

Matto, C. (1994). Aves sin nido. Venezuela: Biblioteca Ayacucho.

Meléndez, C. (1961). La novela indianista en Hispanoamérica (1832-1889) Río
Piedras: Ediciones de la Universidad de Puerto Rico.

Pereira, A. (2014). Utopia e agonia: o indianismo de Gonçalves Dias. Natal, Brasil: EDUFRN.

Portela, C. (2011). Para além do "caráter ou qualidade de indígena": uma história do conceito de indigenismo no Brasil. 274 f. Tese (Doutorado em História). Programa de Pós-Graduação em História, Universidade Federal de Brasília, Brasília.

Rama, A. (1987). Transculturación narrativa en América latina (3 ed.). México: Siglo XXI.

Rodríguez, J. (1990). El indigenismo como proyecto literario: revaloración y nuevas perspectivas. Hispamérica, año XIX(55), 41-50.

Schwartz, J. (2008). Vanguardas Latino-americanas: polêmicas, manifestos e textos críticos. (2 ed.). São Paulo: EDUSP. 